\title{
Analysing the Media Consumer Trends During the COVID-19 Period-The Rise of Otts in India
}

\author{
Sristy Lalaika V, Kalathila Uthej, Shreeya Rishi K
}

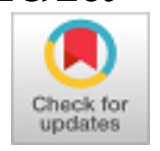

\begin{abstract}
In the wake of an unprecedented COVID-19 widespread, there is a massive development in the media and entertainment industry through OTT (Over-the-Top) platforms which offer video streaming directly to viewers via the internet without cable, broadcast, and satellite television supports. During the self-isolation period, OTTs have increased the overall media consumption to a real high and are now gradually becoming mainstream entertainment destination amidst the growing internet users. This mass intrusion has given an opportunity for many independent platforms to grow and run alongside the likes of already established biggies, Netflix and Amazon Prime. The telecom operators chipped in this aspect and are extensively working on optimizing their operations with advertising revenue and bringing in effective content strategies in order to reach out to more people. The present study focuses on this new development by using the technique of quantitative content analysis in order to know the cause of this sudden increase in OTT channels' viewership among millennials during the lockdown period in India and its impact on traditional modes of communication.
\end{abstract}

Keywords: OTT, Meta medium, Digitization, Chi-square test, Mobile internet penetration, Net neutrality, Quantitative content analysis, Censorship.

Abbreviations and Acronyms

OTT - Over the Top

MSO - Multiple System Operator

DTH - Direct to Home

ANOVA - Analysis of Variance

VoD - Video on Demand

\section{INTRODUCTION}

$W_{\text {ith the advent of new technologies and increasing }}$ levels of internet access, the medium of entertainment has assumed many forms and set new trends in the telecommunication market.

Manuscript received on October 20, 2021.

Revised Manuscript received on October 29, 2021.

Manuscript published on October 30, 2021.

* Correspondence Author

Sristy Lalaika V*, Department of Information Technology, Indira Gandhi Technical University for Women, New Delhi, India.

Email: sristylalaika@gmail.com

Kalathila Uthej, Department of Electronics and Communication Engineering, MVSR Engineering College, Osmania University, Hyderabad (Telangana) India.

Email: uthej2123@gmail.com

Shreeya Rishi K, Department of Electronics and Communication Engineering, MVSR Engineering College, Osmania University, Hyderabad (Telangana) India. Email: shreeyarishi19@gmail.com

(C) The Authors. Published by Blue Eyes Intelligence Engineering and Sciences Publication (BEIESP). This is an open access article under the CC BY-NC-ND license (http://creativecommons.org/licenses/by-nc-nd/4.0/)
The 'Over-the-Top' (OTT) platform is one of them. It is a media service in which the streaming is offered directly to viewers via the internet with no support of cable, broadcast, and satellite television platforms. Earlier, the traditional forms of entertainment have used only linear methods within the confines of a particular space and time and acted as a controller or distributor of such content. Now with the launch of OTT applications, the field of entertainment has witnessed a rapid expansion in the streaming media service and every form of entertainment is recreated anew by it providing a new orientation for telecommunication growth and allowing it to reshape several facets of its market, in terms of resources, for health, transportation, education, agriculture, government and other financial services. In short, the OTT media service platform has now become an indispensable medium of entertainment and has a tremendous influence on the young minds across the world. It has gained immense popularity in India too for the last few years. Since the OTT platform has now become the new normal in the telecommunication market, it has turned the tables for many telecom operators by fuelling rapid advancements in the direction of using digital gadgets and also increased the adaptation of the internet services because unlike the linear fashion trends of traditional television, the over-the-top platform has now enabled customers to easily access content anywhere, anytime in a single go, making more and more people switch over to OTTs. The Jio digitization wave in India is the best example of this development. In addition to this, COVID-19 has played its role. Ever since its outspread, people have started exploring all kinds of virtual means to cater to their everyday needs. This has led to a rising trend among the OTTs. Many production houses have become busy trying to connect to their audiences by releasing their movies/shows on these platforms owing to the nationwide closure of multiplexes and cinema halls. As of now, with its freemium features, Disney+ Hotstar is ruling the hearts of a roaring 400 million Indians followed by Amazon Prime, Netflix, BigFlix, ZEE5, VooT, Sony Liv, Eros Now and other independent content creators like TVF, Spuul, Scoopwhoop, Zoom Studios and Arré. The OTTs have opened up whole new vistas for content creators and in recent times, a huge surge has been seen in their consumer trends. Thus, research in this field on consumer turnouts before and after COVID-19 would enable OTT players to understand and reinvent their strategies accordingly. The importance of OTTs and its impact on millennial consumers before the spread of COVID-19 have been amply dealt with in many research papers.
Blue Eyes Intelligence Engineering and Sciences Publication (BEIESP) (C) Copyright: All rights reserved.

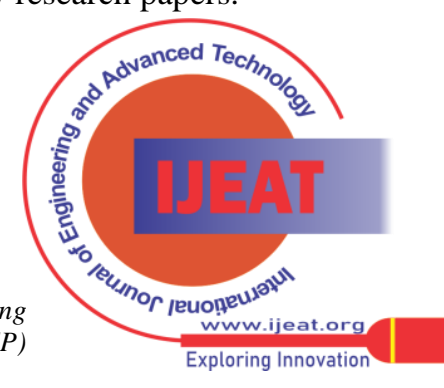


But only a few research papers have focussed on the importance of OTTs after the outbreak of COVID-19. More research needs to be done on how far digitisation has evolved and how the consumer trends have changed overtime ever since the outbreak of COVID-19. This paper takes in a bigger dataset of people and deals with these aspects of the OTTs and also focuses on its impact on the behavioural traits of people from all walks of life across India.

\section{LITERATURE REVIEW}

Keeping in view, the consumer's preference of a meta-medium OTT over a traditional TV, Dr. Sabyasachi Dasgupta and Dr. Priya Grover [1] conducted a small survey to understand the basic factors that led to the adoption of OTT platforms and also its consequences on a long-term basis. The survey methodology consists of a meagre 35 participants within the age limit of 18-30 years coming from in and around the city parts of Maharashtra. While not many studies mentioned a clear insight of the statistics involved in the responses of consumers, this study emphasised solely on them. Based on the responses given, the research points out the net fallout rate of subscribers due to the impact of considerably higher pricing strategies being involved in few OTT subscriptions and telecom service providers. It also stresses on the usage of engaging content based on localisation strategy for enhancing the subscriber turnout rate and to garner more views in a short span of time.

Rahul Sharma and Amit Kakkar [3] in their research study focus on how the Content type, Payment model, Content nature and Language play a major role as product attributes in influencing the adoption of Video on Demand (VoD) services in an Indian scenario. The study shows the relative importance of various product/service attributes and the levels attached with each attribute based on the responses taken from a set of 140 North Indian urban residents by using a Conjoint analysis. The study concludes that the consumers are more likely to opt for cost effective VoDs followed by the content nature, content type and language. Gimpel.G [2] in his paper focuses on the growth of the telecom industry with regard to content consumption. On the basis of platform theory, with the help of data collected from 22 senior executives, he concludes that OTT platforms "produce original content and work on licensed agreements." He also proposes the idea of diversifying media in production companies for the widening of revenue portfolios. He also identifies the consumers' preference between OTT firms and MSOs (Multiple System Operators) and dives deeper into theoretical and practical challenges that incur in the marketing strategies of the industry. The research of Prof. Ripal Madhani and Dr. Vidya Nakhate addresses the comparative study of viewers' behaviour over Traditional Television Channels and OTT Video Platforms in Maharashtra [5]. By using a structured questionnaire, the paper studies the behavioural traits of traditional and OTT viewers for a total of 110 people. Later, after examining the OTT viewership among the respondents, the report states that $45 \%$ of viewers spend nearly 1 to 3 hours and another $25 \%$ of them spend more than 3 hours on a daily basis. Also, the paper affirms that when the shift of viewers from television channels to OTT platforms are correlated, the correlation

coefficient is 0.902 . Furthermore, it also proves that different age groups prefer different video platforms by performing a Chi-square test. In conclusion, the study indicates that viewers favour OTT platforms over television channels as they provide convenience of time and place, cheaper data accessibility and access to quality content in minutes of time. The study of Muhammad Farooq and Valliappan Raju explains the impact of OTT services on the Telecom Companies in the Era of Transformative Marketing [6]. This paper concentrates on the revenue loss for telecom customary voice and informing sectors due to the induction of OTT services like WhatsApp, Messenger, Telegram etc. that are provided with many new features like sharing pictures, videos, file transferring, video calling, group conversations etc. The study also states that customers are purchasing fixed web plans and are utilizing OTT for correspondence which is ripping apart the voice and SMS income of telecom organizations. The research specifies that OTT services at one point of time were the sole reasons for decrease in revenue but on the greener side, they also created favourable situations for telecom companies to earn from internet data plans. The paper concludes that soon with the launch of 5G, the internet speed will increase up to 10Gbps and the telecom organizations in turn will have to update and expand their infrastructure.

The research work of Manoj Kumar Patel [7] reveals that the OTT platforms are evolving and are having a considerable edge over the traditional mediums of entertainment. By conducting an online survey with 95\% of the major stakeholders being aged between 20 to 40 years, the study states that the growth of OTT will definitely increase in India because of its cost effectiveness and the consistent evolution of content richness. The author finally concludes that the rapid growth of streaming media in India is due to the liberty it provides for the consumers to access content anywhere irrespective of time.

The research conducted by Sujatha Joshi [9] mainly focuses on analysing the change in consumer trends in India with the emergence of OTT platforms and its effect on the traditional platforms. According to the analysis conducted in the paper, the author says that cost, content availability, net neutrality and user friendliness are the game changing factors as OTT platforms offer all those at a reasonable price when compared to traditional ones. The paper states that the main advantage of OTT players is that they can use the telecom infrastructure without even paying for it and they are not subjected to the regulatory regimes that apply to the traditional services.

The paper concludes that the main drawback of the traditional platform operators is that they are constrained by the geographical boundaries whereas OTT players are not constrained and can provide consumer services throughout the world regardless of any geographical boundaries. The paper also shares few creative strategies for the upliftment of telecom operators and traditional platforms to boost their economical game and give equal competition to the OTT platforms. According to the research of Baldry et.al.

Published By:

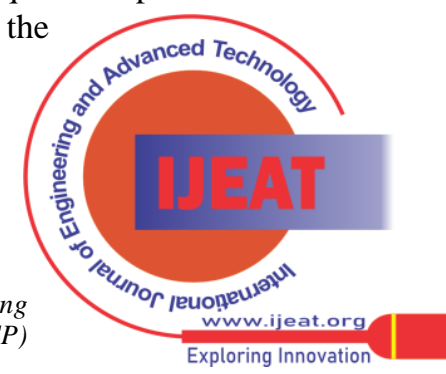


[12], changes are being made by traditional application providers to tune in to the market demands. The paper addresses the fact that the emergence of OTT providers into the market has brought about a sea change in the network operators' competitive situation. It also states that the hands of the operators are tied down because of the regulatory obligations imposed on them and recommends few adaptations to the rules which have been impeding the operators' attempts to respond to the competitive challenges they face. According to Rohit Jacob Jose [13], one of the most recent shifts in the way people are consuming media content is the shift from DTH (Direct to Home) to OTT platforms. The paper revolves around the primary data collected from an online survey having 103 respondents. The paper uses One-way ANOVA (Analysis of Variance) method to understand the two significant factors, user friendliness and content richness which influenced the viewers shifting from DTH to OTT platforms in India. This study also highlights the factors on how OTT platforms can focus their efforts on improving their customer satisfaction.

\section{METHODOLOGY}

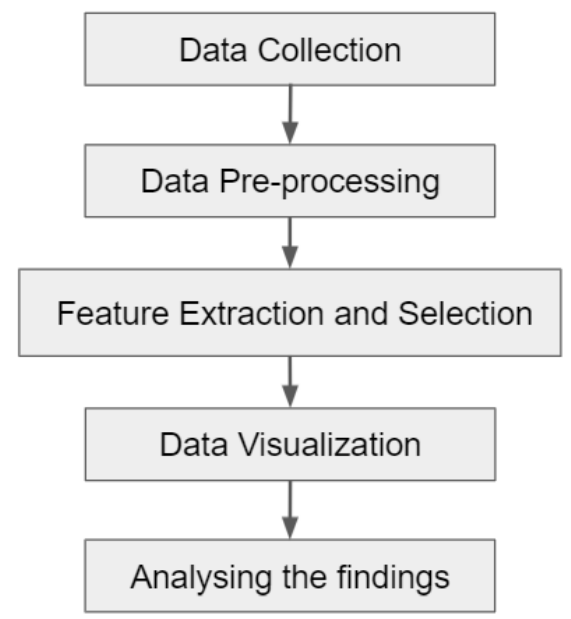

Fig. 1. Step-by-step methodology chart

This research uses Quantitative content analysis in order to understand the systematically categorized textual, visual, or aural variables. Quantitative content analysis [15] is used in different fields but is majorly deployed in the communication sectors (Kevin Coe, Joshua M. Scacco 2017). Here, the percentage analysis is one of the essential statistical tools used for the interpretation of source data. It basically deals with the number of respondent's responses to a particular question whose percentage arrives through population as a whole that is selected for the study. The online survey for this study was active from Sep 24, 2020 to Oct 7, 2020 on all social media platforms and garnered a total of 491 responses who were specifically aged between $18-30$ years.

\section{RESEARCH QUESTIONS}

In accordance with the research firm Nielsen, the overall media consumption during the lockdown period has increased by 60 percent and due the rapid increase in demand and reach, it is clear that it has remarkable impact on traditional modes of entertainment. In this study, the following research questions have been formulated to understand OTT viewership preferences and watching habits of consumers before and after the wake of pandemic in India.

1. How many hours a consumer spends on the OTT platforms before and after the onset of COVID-19?

2. Which OTT platforms the consumer prefers to watch?

3. At what time and with whom would the consumer prefer to watch?

4. How much is the consumer willing to pay for an OTT platform?

5. What are the factors that caused due to the rise in OTT channels viewership before and during lockdown period in India?

6. If not OTT platforms, what would the consumer choose and why?

\section{ANALYSIS AND FINDINGS}

The purpose of this research is to find out the views and preferences of people with regard to OTT watching. The study has a sample size of 491 respondents ( $52.55 \%$ females and $47.45 \%$ males) with $93.08 \%$ of the respondents ranging in the age groups of 18-30 from different parts of India.

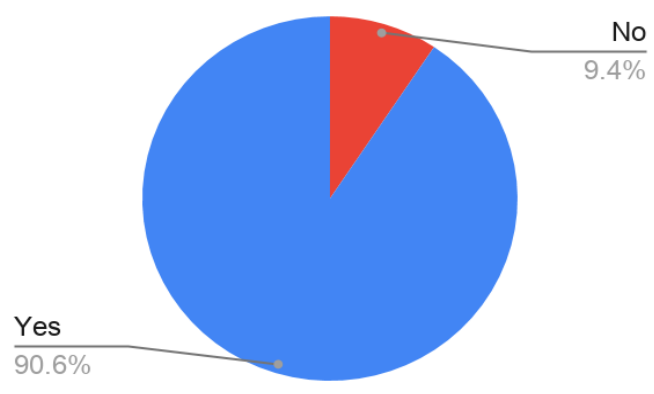

Fig. 2. OTT vs. Traditional platforms

Fig.2 shows the percentage of people who prefer OTTs to Traditional platforms. It can be seen that $90.6 \%$ of the respondents from the collected sample use OTT platforms and the remaining $9.4 \%$ people still prefer traditional platforms over OTTs. When asked, people stated that the higher pricing strategy of OTTs is one of the main reasons for them to back off the ladder.

Few others are preferring third party applications that stream the same content illegally (without copyrights).

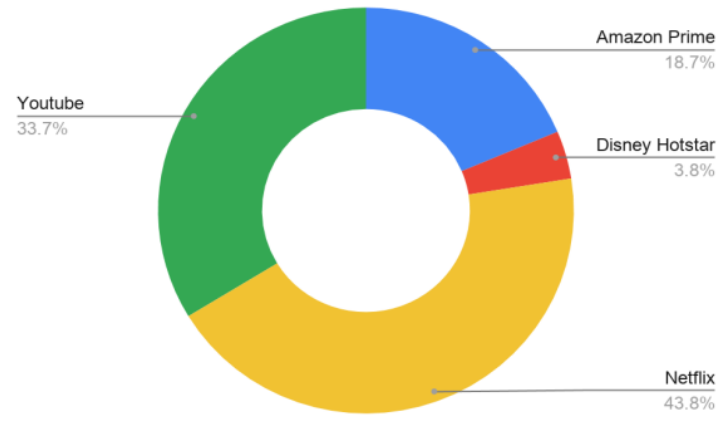

Fig. 3.Most preferred OTTs

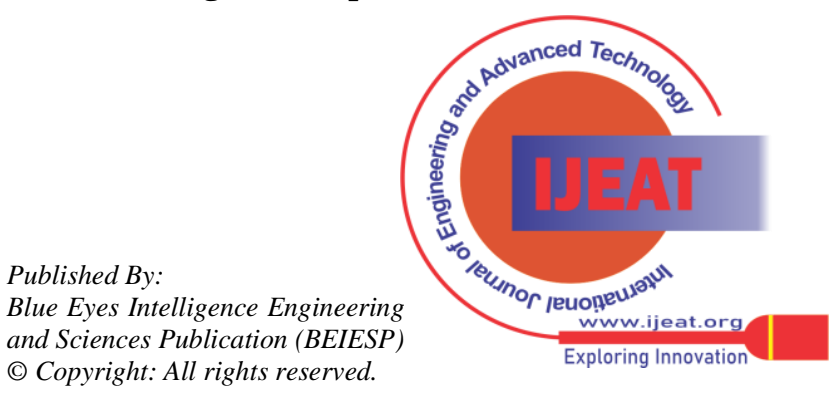


Fig. 3 shows the percentage of the most preferred OTTs by the respondents. Netflix plays it cool by being the highest of all with $43.8 \%$ viewer base. YouTube, being the second highest has $33.7 \%$ followed by Amazon prime with $18.7 \%$ and Disney Hotstar with 3.8\%. When asked about the reason for choosing Netflix and YouTube over others, few stated that the user interface of Prime is not friendly and even after having freemium services, the content in Hotstar is not highly enriched. Fig.4 interprets how the most preferred OTTs differ with respect to qualification. Here, we can clearly see that the subscription plans have a great influence on the selection of OTTs. The grade of pay is directly proportional to the most preferred OTT. YouTube is the go-to platform among high school students as its subscription plans are a bit lesser.

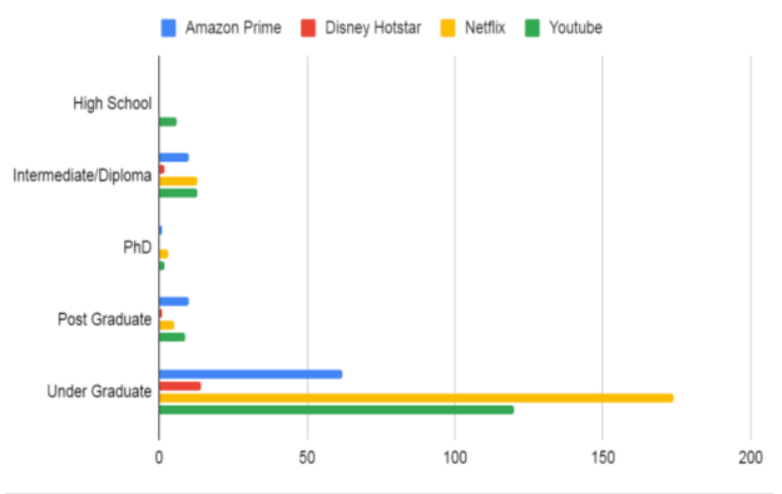

Fig. 4.Qualification vs. Most preferred OTT

Under the Intermediate/Diploma column, Netflix and YouTube are the most preferred OTTs followed by Amazon Prime and Disney Hotstar.

For PhD respondents, Netflix is followed by YouTube and Amazon Prime. Under the Postgraduate column, Amazon Prime is the most preferred OTT followed by YouTube, Netflix and Disney Hotstar. For Undergraduates, Netflix is the most preferred OTT followed by YouTube, Amazon Prime and Disney Hotstar.

Specifically, when students were asked about why they chose OTT over Traditional TV, most of them responded that streaming platforms help them academically and personally like relieving stress and anxiety after a hard day at work. Few others stated that OTTs also help them in fighting down loneliness and boredom.

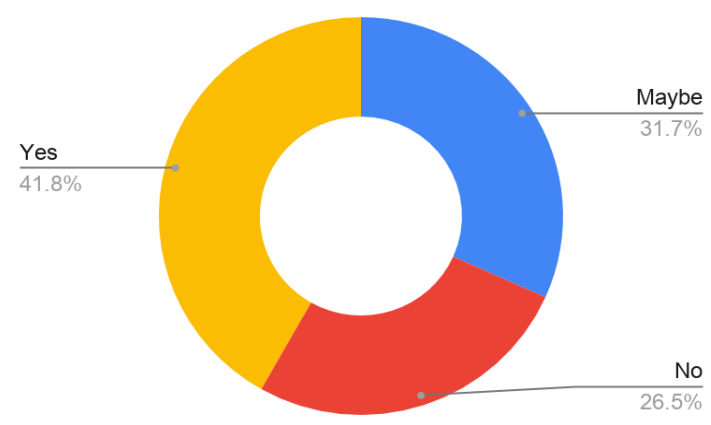

Fig. 5.Do you binge watch?

Fig. 5 shows the binge watch ratio before and after the COVID-19 in which a major chunk of the audience who voted Yes (41.8\%) are lying under the age group of 18-23 years.
The spike in the binge watch ratio is mostly observed among the millennials because television shows unlike OTTs are not novel or innovative and stream only conventional sagas that revolve all year round.

OTTs on the other hand have come up with new storylines and screen plays and release all of the content for a specific show/series within a day because of which people are sliding over to binge watching shows all night. In general, most of the audience crave for thrilling and engaging content and this cannot be satiated by conventional television programs. Hence, millennials are moving towards OTTs as they offer a wide variety of genres ranging between all regional contexts which are relevant, contemporary and binge worthy.

The other percentage of respondents who voted for Maybe (31.7\%) stated that binge watching shows would depend on how exciting and thrilling the show is.

The No (26.5\%) category has older generation consumers ranging between $30-50$ years.

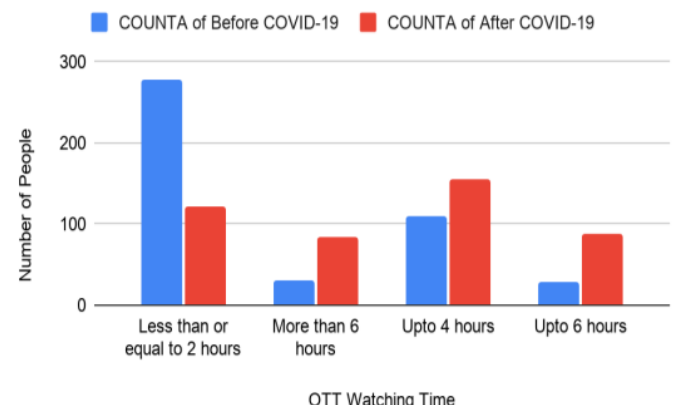

Fig. 6.Before COVID19 vs. After COVID19

Fig.6 shows the OTT Watching time of respondents before and after the onset of COVID-19. In regard to our question on how much time they spend on OTT platforms before and after the COVID-19 outbreak, the viewers' responses are shown in the following table.

Table- I: Watch time of the respondents

\begin{tabular}{|c|c|c|}
\hline Watch Time & Before COVID-19 & $\begin{array}{c}\text { After } \\
\text { COVID-19 }\end{array}$ \\
\hline More than 6 hours & $6.7 \%$ & $18.7 \%$ \\
\hline Upto 6 hours & $6.5 \%$ & $19.6 \%$ \\
\hline Upto 4 hours & $24.5 \%$ & $34.6 \%$ \\
\hline $\begin{array}{c}\text { Less than or equal to 2 } \\
\text { hours }\end{array}$ & $62.2 \%$ & $27.2 \%$ \\
\hline
\end{tabular}

Table-I shows that $6.5 \%$ of the respondents whose watch time was upto 6 hours before COVID-19 increased to 19.6\% after the onset of COVID-19. Also, there is a massive decline in the two hours watch time column. Four hours watch time column is standing steady before and after COVID-19. Fig.7 shows the analysis of gender against their preference of watching (watching alone/family/friends). In both male and female categories, watching alone bar is highly set. When asked about the reasons, a few of them responded saying that their focus will be deterred with people around and that they are afraid of being judged for the content they watch.

Published By:

Blue Eyes Intelligence Engineering

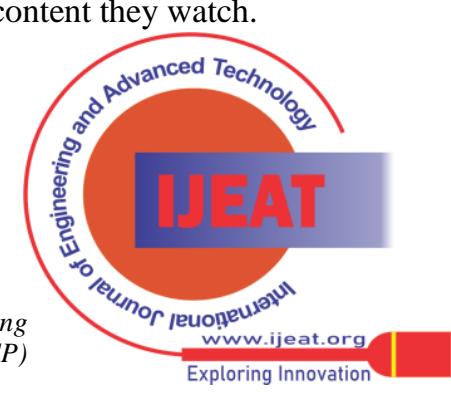


By this research method, we have understood the viewer's choice of preference on whether they prefer OTT platforms or the traditional television from our first question and according to the preferences they chose, the analysis has been done which highlighted the spike in viewership before and after the COVID-19 outbreak. Overall, there is $15-20 \%$ rise in the viewership and the watch time has dramatically increased as people are staying home owing to the rise of COVID-19 cases in recent times. OTT platforms have been

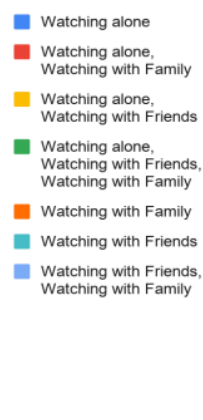

Fig. 7. Gender vs. Watching preference

trying to mark a place for themselves in the Indian market and this outbreak of COVID-19 has come as a blessing in disguise, pushing the viewer base to a skyrocket high. Consumers crave for variety in the content and that is being fulfilled by almost all OTT players. Furthermore, this lockdown has made people realise how comfortable and convenient OTT platforms are.

\section{CONCLUSION}

The data collected by a structured questionnaire has been analyzed and the results are interpreted in the form of pie charts and bar graphs. The figures show that there is a significant difference in the watch time of OTT platforms before COVID-19 and after COVID-19 (during lockdown). With the developing change in the telecommunication industry, a massive surge is seen in the use of OTT platforms due to the easy access of internet (4G) at minimum costs. The extensive ability to choose one's favorite content at one's own convenience is the main reason for the consumers to prefer OTT platforms to traditional mediums. Also, Traditional TVs not being user friendly and being limited to conventional dramas have helped a lot in spurring the growth of contemporary OTTs. When the change from $4 \mathrm{G}$ to $5 \mathrm{G}$ in the telecommunication industry takes place, the internet speed will increase to $10 \mathrm{Gbps}$ which will benefit the OTT platforms in providing content with higher resolution (like $4 \mathrm{~K}$ or $8 \mathrm{~K})$. OTTs are trying to satiate consumer's needs slowly and steadily and in the next few years with technological advancements round the corner, OTTs will become a go-to mode for people in India because of its ease to access enriched content at simpler costs anywhere, anytime on a single click. And imposing a complete censorship on such content will transform it into the same old mainstream cinema and television shows. And thus, it will lead to an increase in the amount of piracy cases and in turn pose a hindrance to the premium subscriber base of OTTs. Besides, it will also undermine the creative freedom of content creators as they will have to circumnavigate according to the censorship norms.

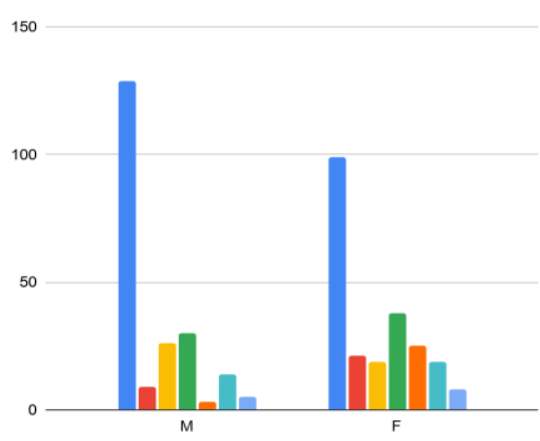

\section{ACKNOWLEDGMENT}

This paper and the research behind it would not have been possible without the exceptional support of our supervisor $\mathrm{K}$ Krishna Chaitanya Rao and the extended guidance from Publishsutra and Mahawiki communities.

\section{REFERENCES}

1. Dr. Sabyasachi Dasgupta and Dr. Priya Grover, Understanding Adoption Factors of Over-the-Top Video Services among Millennial Consumers, International Journal of Computer Engineering and Technology, 10(1), (2019), $\quad$ pp. $\quad 61-\quad 71$. http://www.iaeme.com/IJCET/issues.asp?JType=IJCET\&VType=10\&I Type $=1$

2. Gregory Gimpel (2015) The Future of Video Platforms: Key Questions Shaping the TV and Video Industry, International Journal on Media Management, 17:1, 25-46, DOI: 10.1080/14241277.2015.1014039 (2015).

3. Rahul Sharma, Amit Kakkar, A Conjoint-Based Approach To Consumer Preferences in VoD Services, International Journal of Engineering and Advanced Technology (IJEAT) ISSN: 2249-8958, Volume-8 Issue-4C, April 2019.

4. KPMG on India's Media and Entertainment report (2019) Retrieved Aug. $\quad 30, \quad 2020$ from https://assets.kpmg/content/dam/kpmg/in/pdf/2019/08/india-media-ente rtainment-report-2019.pdf

5. Prof. Ripal Madhani, Dr. Vidya Nakhate. (2020). Comparative Study of Viewers' Behaviour Over Traditional Television Channels And Ove Ott Video Platforms In Maharashtra. International Journal of Advanced Science and Technology, 29(12s), 2076-2086. Retrieved from http://sersc.org/journals/index.php/IJAST/article/view/24371

6. Farooq, Muhammad \& Raju, Valliappan. (2019). Impact of Over-the-Top (OTT) Services on the Telecom Companies in the Era of Transformative Marketing. Global Journal of Flexible Systems Management. 20. 177-188. 10.1007/s40171-019-00209-6.

7. Manoj Kumar Patel, Rahul Khadia, Dr. Gajendra Awasya A Study: OTT Viewership in "Lockdown" and Viewer's Dynamic Watching Experience International Journal on Transformations of Media, Journalism \& Mass Communication Vol. 5, Issue 2-2020 ISSN: 2581-3439.

8. $55 \%$ of Indians prefer OTT platforms vs $41 \%$ that still prefer DTH MoMAGIC survey Retrieved Aug. 30, 2020 from https://brandequity.economictimes.indiatimes.com/news/media/55-of-i ndians-prefer-ott-platforms-vs-41-that-still-prefer-dth-momagic-survey/ 70858815

9. Joshi, Sujata \& Sohag, Sarkar \& Dewan, Tanu \& Chintan, Dharmani \& Shubham, Purohit \& Sumit, Gandhi. (2015). Impact of Over the Top (OTT) Services on Telecom Service Providers. Indian Journal of Science and Technology. 8. 145. 10.17485/ijst/2015/v8iS4/62238

10. KPMG on How OTT platforms can remain 'on-demand ready' (2019) Retrieved Aug. $\quad 30, \quad 2020$ from http://ficci.in/events/23490/ISP/The\%20Digital\%20First\%20journey_ Web.pdf

11. Baldry, Shirley \& Steingröver, Markus \& Hessler, Markus A., 2014 "The rise of OTT players: What is the appropriate regulatory response?," 25th European Regional ITS Conference, Brussels 2014101398 International Telecommunications Society (ITS).

12. Rohit Jacob Jose, Factors influencing the shift from traditional TV to OTT platforms in India (2020) International Journal of Advanced Science and Technology Vol. 29, No. 7s, (2020), pp. 4044-4051.

13. Internet usage in India - statistics \& facts by Sandhya Keelery (2020) Retrieved Aug. 30, 2020 from https://www.statista.com/topics/2157/internet-usage-in-india/

14. Coe, K. and Scacco, J.M. (2017). Content Analysis, Quantitative. In The International Encyclopedia of Communication Research Methods (eds J. Matthes, C.S. Davis and R.F. Potter). doi:10.1002/9781118901731.iecrm0045.

15. How COVID-19 increased viewers' appetite for OTT content by Priyadarshini Patwa, Features Editor, Entrepreneur India (2020)

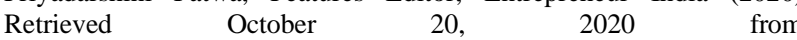
https://www.entrepreneur.com/article/350318

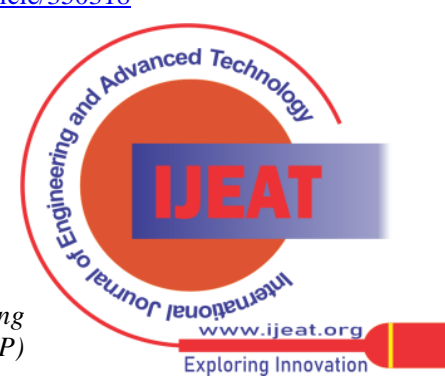




\section{AUTHORS PROFILE}

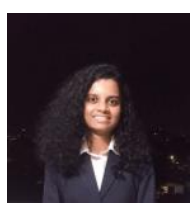

Sristy Lalaika V, is an avid reader, blogger, content writer and currently pursuing her M.Tech in Cyber Security at Indira Gandhi Delhi Technical University for Women, New Delhi. Her research interests include Data Analytics, Cryptography and Information Security. She has actively volunteered for various Cyber awareness camps. Her recent works include analyzing the behavioral traits of stressed individuals and its aftermath effects on CRP rate by using Machine learning algorithms, and optimizing casting process parameters by using DoE techniques. Her current research activities are directed towards Cyber Forensics and Security in Next Generation Networks.

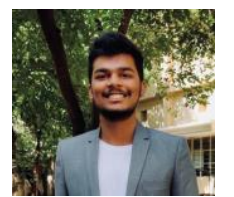

Kalathila Uthej, is a Bachelor of Engineering student, pursuing his final year in Electronics and Communications at MVSR Engineering College, Osmania University. His research interests incorporate the fundamentals of research and design thinking, data analytics, Natural language processing, Machine learning and Internet of Things. He persistently worked on projects whose objectives include the concepts of NLP model for visually challenged, Smart Security System using IOT, Image Compression and Quick Response scanning system.

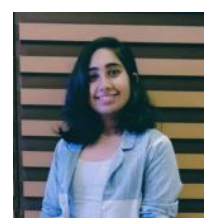

Shreeya Rishi K, is a passionate researcher pursuing her Bachelor of Engineering in Electronics and Communication Engineering at MVSR Engineering College, Osmania University. Her research interest comprehends in the field of Computer vision, Machine Learning and Data analytics. Recently, she worked on the projects which include face recognition using HAAR cascade, brain tumor segmentation using K-Means algorithm, Image enhancement using median filter.

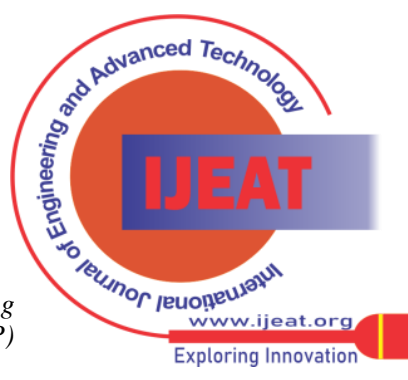

